\title{
Daily intake of fermented milk with Lactobacillus casei strain Shirota reduces the incidence and duration of upper respiratory tract infections in healthy middle-aged office workers
}

\author{
Kan Shida ${ }^{1}$ Tadashi Sato ${ }^{1}$ Ryoko Iizuka ${ }^{1}$ Ryotaro Hoshi ${ }^{2}$ Osamu Watanabe ${ }^{2}$ \\ Tomoki Igarashi $^{2} \cdot$ Kouji Miyazaki $^{1} \cdot$ Masanobu Nanno $^{1} \cdot$ Fumiyasu Ishikawa $^{1}$
}

Received: 10 June 2015 / Accepted: 23 September 2015 / Published online: 29 September 2015

(C) The Author(s) 2015. This article is published with open access at Springerlink.com

\begin{abstract}
Purpose Although several studies have demonstrated the efficacy of probiotics for preventing upper respiratory tract infections (URTIs) in at-risk populations, including children and the elderly, few studies have investigated the efficacy of probiotics in healthy adults living normal, everyday lives. Thus, we tried to evaluate the effects of Lactobacillus casei strain Shirota-fermented milk (LcS-FM) on the incidence of URTIs in healthy middle-aged office workers.

Methods In a randomized controlled trial, 96 eligible male workers aged 30-49 years consumed LcS-FM containing $1.0 \times 10^{11}$ viable $\mathrm{LcS}$ cells or control milk $(\mathrm{CM})$ once daily for 12 weeks during the winter season. URTI episodes were evaluated by a physician via a questionnaire of URTI symptoms.

Results The incidence of URTIs during the intervention period was significantly lower in the LcS-FM group than in the CM group (22.4 vs. $53.2 \%, P=0.002)$. The timeto-event analysis showed that the LcS-FM group had a significantly higher URTI-free rate than the CM group over the test period (log-rank test: $\chi^{2} 11.25, P=0.0008$ ). The cumulative number of URTI episodes and cumulative days with URTI symptoms per person was lower in the LcS-FM group, and the duration per episode was shorter. Inhibition of both reductions in NK cell activity in peripheral blood
\end{abstract}

Kan Shida, Tadashi Sato, and Ryoko Iizuka have contributed equally to this work.

Kan Shida

kan-shida@yakult.co.jp

1 Yakult Central Institute, 5-11 Izumi, Kunitachi-shi, Tokyo 186-8650, Japan

2 Faculty of Research and Development, Yakult Honsha, Shinbashi, Tokyo 105-8660, Japan mononuclear cells and increases in salivary cortisol levels was observed in the LcS-FM group.

Conclusion The results suggest that the daily intake of fermented milk with LcS may reduce the risk of URTIs in healthy middle-aged office workers, probably through modulation of the immune system.

Keywords Probiotics - Lactobacillus casei strain Shirota - Upper respiratory tract infection · Common cold . NK cell activity $\cdot$ Cortisol

\section{Introduction}

The human intestinal tract harbors more than 100 trillion bacteria, and the commensal gut microbiota plays a pivotal role in maintaining the health of the host [1]. Thus, much attention has been given to probiotics, which can survive the intestinal tract and recover/maintain balanced gut microbiota when ingested orally $[2,3]$. Some strains of lactobacilli and bifidobacteria are popular probiotics, usually consumed as fermented dairy products or supplements [4]. Among the health benefits of probiotics, the prevention or control of infectious diseases is one of the most promising targets $[5,6]$. Several studies have demonstrated that some probiotics are effective against not only infections in the gastrointestinal tract, but also those in the respiratory tract $[7,8]$.

Many clinical trials against upper respiratory tract infections (URTIs), such as the common cold and influenza, have evaluated various probiotic strains, and many of these have demonstrated efficacy against URTIs [7, 8]. The target populations in these clinical trials have generally been infants, children, students, and the elderly [9-12]. Since the immune defenses in these populations are relatively 
weak, the use of probiotics might be pertinent. However, few studies have investigated the efficacy of probiotics in healthy adults $[13,14]$.

Maintaining the immune defense system within a normal healthy state lowers the risk of URTIs. NK cell activity and salivary immunoglobulin A (IgA) are considered important in the prevention of URTIs $[15,16]$. However, several environmental factors, including a stressful lifestyle, are likely to weaken the immune defense system [17], which may result in an increase in the risk of URTIs. There is some evidence to suggest that some probiotic strains restore NK cell activity and salivary IgA levels [18-20]. Thus, daily ingestion of a probiotic beverage might maintain normal immune function and control URTIs.

Lactobacillus casei strain Shirota (LcS) is a probiotic that can survive within the intestinal tract and recover balanced gut microbiota [21]. The immunomodulatory activities of the strain have been studied extensively in animal models and human studies [18, 22]. Studies in healthy subjects with low NK cell activity showed that ingestion of LcS-fermented milk (LcS-FM) recovered the activity [18]. Clinical trials have shown that $\mathrm{LcS}$ effectively reduces the risk of bladder cancer and colorectal tumor recurrences, and it has been proposed that immunomodulatory activities, including the recovery of NK cell activity, are among the underlying mechanisms [23]. Therefore, accumulating evidence implies that this probiotic has potential use as an immunostimulatory food material.

Some clinical trials with LcS have targeted URTIs. A double-blind, randomized, placebo-controlled trial in healthy elderly people attending day care facilities showed that consumption of LcS-FM reduced the duration of each URTI episode, but not the incidence rate [24]. Another trial conducted in healthy elderly nursing home residents showed a nonsignificant decreasing trend in the incidence of upper respiratory symptoms via LcS-FM ingestion [25]. On the other hand, a study conducted among healthy young athletes of endurance-based sports showed that $\mathrm{LcS}$ was effective in preventing URTIs [20]. In the latter study, daily consumption of LcS-FM significantly reduced the incidence of URTIs and the cumulative number of URTI episodes. Variations in the efficacy of LcS-FM might be due to differences among study populations.

We think that it is important to evaluate the efficacy of LcS in normal healthy adults, because events in daily life can lead to weakened immune defense responses. Thus, healthy middle-aged office workers were selected for this study. We measured the effect of the daily consumption of LcS-FM on the incidence of URTIs as the primary outcome. Additionally, we analyzed both NK cell activity and salivary IgA levels as immunological markers, and the levels of cortisol in saliva as a stress marker.

\section{Materials and methods}

\section{Participants}

Healthy male workers living in Tokyo or its suburbs, aged 30-49 years, and working within office buildings were recruited for this study via Web site advertising. The exclusion criteria were as follows: (1) working outside the office building twice or more a week; (2) difficulty providing saliva and blood samples; (3) pollinosis, chronic rhinitis, asthma, or milk allergy; (4) periodontitis or gingivitis; (5) history of serious liver, kidney, heart, lung, or gut disease; (6) receiving current medical treatment; (7) regularly consuming probiotics or fermented milk; (8) taking drugs or supplements that might affect the outcome of the study; (9) history of influenza vaccination or infection within the last 6 months; and (10) being deemed ineligible for this study by a physician, based on blood chemistry, blood pressure, pulse rate, or other reasons.

Participants were given a detailed explanation of the purpose and potential risks of the study, and 217 workers provided written consent and participated in a screening test, in which blood, urine, and saliva analysis, a physical examination, measurements of blood pressure and pulse, and a questionnaire regarding lifestyle and working profile were performed. Seventy-eight participants met the exclusion criteria, 10 with salivary IgA concentrations of $509 \mu \mathrm{g} / \mathrm{ml}$ or more were excluded, and 29 declined to participate prior to allocation. The remaining 100 participants were randomly allocated to two groups: the LcS-FM group and the control milk (CM) group.

\section{Sample size calculation}

The sample size was calculated by assuming that the incidence of URTIs during the test period of 12 weeks would be $80 \%$ and LcS-FM would reduce the incidence by $35 \%$ $[13,20,26]$. At a significance level of 0.05 with $80 \%$ power, it was estimated that 44 participants were needed per group to detect significant differences between the groups by the Chi-square test. Thus, 100 participants were included in this study.

\section{Study design}

We conducted a randomized, controlled trial from December 8, 2012, through March 5, 2013, at the Chiyoda Paramedical Care Clinic (Tokyo, Japan). The study was conducted according to the guidelines laid down in the Declaration of Helsinki. All procedures were approved by the Institutional Review Board of the Chiyoda Paramedical Care Clinic. Written, informed consent was obtained from all participants. 


\section{Test drinks}

LcS-FM consisted of skimmed milk, high-fructose corn syrup, sugar, flavoring, and a minimum of $1.0 \times 10^{11}$ live LcS cells. Probiotic strain LcS (YIT 9029) was obtained from the Culture Collection Research Laboratory of the Yakult Central Institute. Milk was used as a control beverage [12]. LcS-FM and CM drinks were placed into plastic bottles. The test drinks were delivered to each participant once a week under refrigeration and stored in a refrigerator until consumption.

\section{Procedures}

Participants were allocated randomly to either the LcSFM or CM group. Both groups were asked to consume one bottle of the test drink every day for 12 weeks and to refrain from consuming any other probiotic foods and supplements.

Participants kept a health diary during the intervention period each day before bedtime, recording body temperature, any symptoms of illness, and the impact of any symptoms on their daily activities by rating the impact as none/ light, moderate, or severe. The test drinks consumed and all prohibited foods and supplements were also recorded in the diary. Participants were also asked to record possible symptoms of URTIs on the URTI symptom questionnaire on a daily basis.

Participants visited the clinic at weeks 0,6 , and 12 during the intervention period, and saliva and blood samples were collected. Participants were free to consult any doctor of their choice when experiencing symptoms of an illness, but were required to record the details of any diagnosis provided, hospital care given, prescribed and non-prescribed medications taken, and the results of influenza virus tests via influenza diagnosis kits if applicable in the health diary.

\section{Evaluation of URTIs}

URTI episodes were evaluated by a physician at the clinic based on the information recorded in the health diary, the URTI symptom questionnaire, and interviews at 6 and 12 weeks after the intervention period commenced [12, 20]. The URTI symptom questionnaire consisted of questions on the following 16 symptoms: (1) fever; (2) chill; (3) headache; (4) runny nose; (5) stuffy nose; (6) sneezing; (7) cough; (8) sore throat; (9) sputum; (10) malaise; (11) muscular pain; (12) joint pain; (13) nausea; (14) diarrhea; (15) stomach ache; and (16) itchy eyes. The non-numerical ratings of none/light, moderate, and severe were scored as 1,2 , and 3, respectively, for each item. The daily symptom severity score for URTIs was calculated by summing all the scores for each item, except those for nausea, diarrhea, stomach ache, and itchy eyes, which were set to distinguish a URTI episode from gastrointestinal infections and pollinosis. Thus, the maximum score for daily symptom severity was 36 . Symptoms separated by more than 2 consecutive symptom-free days were recorded as separate episodes.

Influenza was diagnosed according to the results of influenza virus test kits conducted as required at any hospital and was counted as a URTI episode. URTIs other than influenza were recorded as the common cold.

\section{NK cell activity}

Peripheral blood mononuclear cells were freshly isolated, and NK cell activity was measured by the chromium release assay using K562 target cells. Peripheral blood mononuclear cells and ${ }^{51} \mathrm{Cr}$-labeled $\mathrm{K} 562$ cells were incubated at a ratio of $20: 1$ for $3.5 \mathrm{~h}$, and radioactivity released from lysed target cells was measured. The percentage of specific lysis was calculated as NK cell activity using the following formula: specific lysis $(\%)=$ (experimental release - spontaneous release)/(maximal release - spontaneous release) $\times 100$.

\section{Saliva analysis}

Saliva was collected between 09:00 and 11:00 at the clinic by the passive drool method. Participants were asked to allow saliva to pool in the mouth for 3 min before transferring it via a straw to a collection tube. Saliva collection was repeated four times with 1-min intervals. The pooled saliva was centrifuged at $1500 \times g$ for $15 \mathrm{~min}$, and the supernatant was stored below $-20{ }^{\circ} \mathrm{C}$ until analysis.

The levels of secretory IgA and cortisol were determined by immunoassay kits (Salimetrics, PA) according to the instruction manuals.

\section{Adverse events}

General biochemical and hematological testing of blood, urinary qualitative examinations, and physiological tests were performed at 0 and 12 weeks, and the results before and after the intervention period were compared. Any adverse health events were recorded by participants in their health diaries during the intervention period and confirmed by the clinic physician at weeks 6 and 12 .

\section{Statistical analyses}

The difference in URTI incidence between the groups was analyzed by the Chi-square test. Time-to-episode curves for the first URTI in the groups were described 


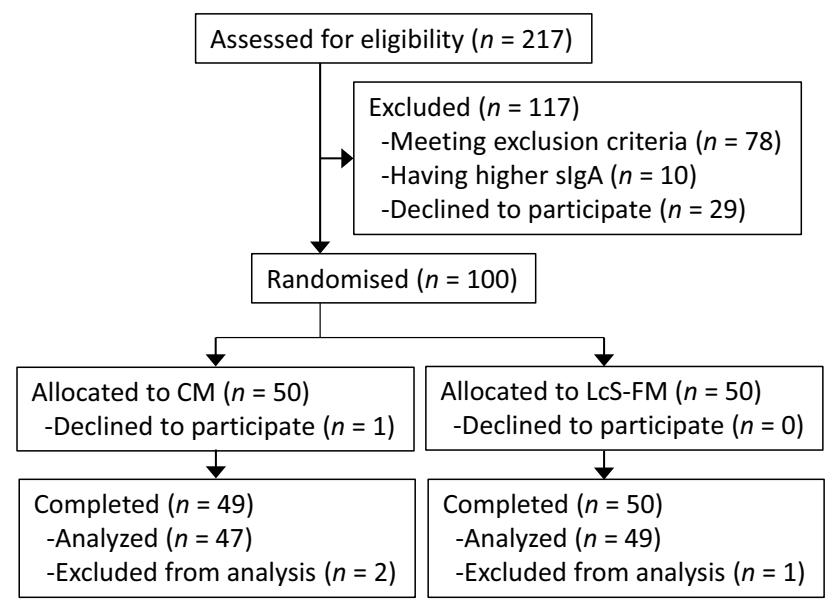

Fig. 1 Participant flow diagram for this study

by the Kaplan-Meier method, and the difference was analyzed by the log-rank test (27). A hazard ratio was estimated using unadjusted Cox proportional hazard model. The risk reduction efficacy was calculated using the following formula: efficacy $(\%)=(1-$ hazard ratio) $\times 100$. Comparisons of the cumulative numbers of URTI episodes per person and levels of symptom severity between the groups were performed by the Wilcoxon two-sample test. Comparisons of cumulative days with symptoms per person and the durations of URTI episodes were achieved using the unpaired Student's $t$ test. Unpaired and paired Student's $t$ tests were, respectively, used for intergroup and intragroup comparisons of NK cell activity, salivary IgA secretion, and cortisol levels. Two-tailed $P$ values $<0.05$ were considered statistically significant. All analyses were performed using IBM SPSS software version 20.0 (IBM Japan, Tokyo, Japan) and SAS software version 8.2 (SAS Institute Japan, Tokyo, Japan).

\section{Results}

\section{Participant flow and characteristics}

The participant flow diagram for this study is shown in Fig. 1. Eligibility was assessed in 217 male workers. Among them, 100 participants were recruited to the study and allocated to either the LcS-FM or CM group. One participant in the CM group did not start the intervention due to personal reasons, and 99 participants completed it. Of the 99 participants, 3 were excluded from the analysis for the following reasons: one started medication for hypertension during the intervention period; one consumed prohibited probiotic drinks on 10 days during the test period; and one was found to meet an exclusion criterion of the study. Thus, 96 participants comprising 49 participants in the LcS-FM group and 47 participants in the CM group were used for data analysis.

The baseline characteristics of the 96 eligible participants from the two groups are summarized in Table 1. No significant differences in age, body mass index, living with a child, smoking habits, or salivary IgA levels were found between the groups. Both groups showed very good compliance in terms of test drink consumption.

\section{Incidence of URTIs}

The primary outcome measure of this study was URTI incidence, the results of which are shown in Table 2. The incidence rates of URTIs during the intervention period (weeks 1-12) were significantly different at 53.2 and $22.4 \%$ for the CM and LcS-FM groups, respectively $(P=0.002)$. The incidence rate of the common cold was significantly lower in the LcSFM group than in the CM group (18.4 vs. $44.7 \%, P=0.005$ ). The incidence of influenza was $10.6 \%$ in the $\mathrm{CM}$ group and $4.1 \%$ in the LcS-FM group, although this difference did not
Table 1 Baseline characteristics of participants

\begin{tabular}{|c|c|c|c|c|c|}
\hline & \multicolumn{2}{|l|}{$\mathrm{CM}(n=47)$} & \multicolumn{2}{|c|}{ LcS-FM $(n=49)$} & \multirow[t]{2}{*}{$P$ value } \\
\hline & Mean (SD) & $n(\%)$ & Mean (SD) & $n(\%)$ & \\
\hline Age (years) & $40.5(5.9)$ & & $40.6(5.3)$ & & $0.931^{\mathrm{b}}$ \\
\hline BMI $\left(\mathrm{kg} / \mathrm{m}^{2}\right)$ & $23.6(2.7)$ & & $22.8(2.8)$ & & $0.184^{\mathrm{b}}$ \\
\hline No. living with a child ${ }^{\mathrm{a}}$ & & $17(36.2)$ & & $19(38.8)$ & $0.792^{\mathrm{c}}$ \\
\hline No. of smokers & & $9(19.1)$ & & $16(32.7)$ & $0.306^{\mathrm{c}}$ \\
\hline SIgA secretion rate $(\mu \mathrm{g} / \mathrm{min})$ & $57.4(23.7)$ & & $50.8(31.0)$ & & $0.245^{\mathrm{b}}$ \\
\hline Product compliance $(\%)$ & $99.7(1.1)$ & & $99.7(0.9)$ & & $0.930^{\mathrm{b}}$ \\
\hline
\end{tabular}

$C M$ control milk, LcS-FM L. casei strain Shirota-fermented milk, BMI body mass index, SIgA salivary immunoglobulin A

\footnotetext{
${ }^{a}$ No. of participants living with one or more children attending elementary/junior high school

b $P$ values analyzed by the unpaired Student's $t$ test

c $P$ values analyzed by the Chi-square test
} 
Table 2 Primary outcomes: incidence of URTIs

\begin{tabular}{lccc}
\hline & $\mathrm{CM}(n=47)$ & $\mathrm{LcS}-\mathrm{FM}(n=49)$ & $P$ value $^{\mathrm{b}}$ \\
& $n^{\mathrm{a}}(\%)$ & $n^{\mathrm{a}}(\%)$ & \\
\hline Whole period (1-12 weeks) & & 0.002 \\
URTIs & $25(53.2)$ & $11(22.4)$ & 0.005 \\
Common cold & $21(44.7)$ & $9(18.4)$ & 0.201 \\
Influenza & $5(10.6)$ & $2(4.1)$ & \\
1st period (1-4 weeks) & & 0.017 \\
URTIs & $11(23.4)$ & $3(6.1)$ & 0.017 \\
Common cold & $11(23.4)$ & $3(6.1)$ & - \\
Influenza & $0(0.0)$ & $0(0.0)$ & 0.049 \\
2nd period (5-8 weeks) & & 0.069 \\
URTIs & $12(25.5)$ & $5(10.2)$ & 0.293 \\
Common cold & $10(21.3)$ & $4(8.2)$ & \\
Influenza & $3(6.4)$ & $1(2.0)$ & 0.705 \\
3rd period (9-12 weeks) & & 0.603 \\
URTIs & $7(14.9)$ & $6(12.2)$ & 0.484 \\
Common cold & $5(10.6)$ & $5(10.2)$ & $1(2.0)$ \\
Influenza & $2(4.3)$ &
\end{tabular}

URTI upper respiratory tract infection, $C M$ control milk, $L c S-F M L$. casei strain Shirota-fermented milk

${ }^{a}$ No. of participants with URTIs/common cold/influenza

b $P$ values analyzed by the Chi-square test; $P<0.05$ is considered statistically significant

reach statistical significance. Time-to-episode curves for the first URTI are shown in Fig. 2. The curve of the LcS-FM group was significantly higher than that of the $\mathrm{CM}$ group over the test period (log-rank test: $\chi^{2} 11.25, P=0.0008$ ). The URTI-free rates were 0.78 (95 \% CI 0.66-0.89) and 0.47 (95\% CI 0.330.61 ) in the LcS-FM group and the CM group, respectively. The hazard ratio was calculated 0.32 (95\% CI 0.16-0.65).

We further analyzed the incidence rates of URTIs during the following three periods: 1st period (weeks 1-4); 2nd period (weeks 5-8); 3rd period (weeks 9-12). As shown in Table 2, the incidence of URTIs was significantly reduced in both the 1 st and the 2 nd periods $(P=0.017$ and $P=0.049$, respectively) but not in the 3 rd period in the LcS-FM group.

\section{Characteristics of URTI episodes}

The effects of the test drinks on the cumulative number of episodes, the cumulative days with symptoms, and the duration and severity of URTIs were analyzed. The cumulative numbers of URTI episodes per person during the intervention period were 0.7 in the $\mathrm{CM}$ group and 0.3 in the LcS-FM group ( $P=0.004$; Table 3$)$. The LcS-FM group had fewer cumulative days with URTI symptoms per person during the test period, and the duration of each URTI episode was shorter compared with the $\mathrm{CM}$ group ( $P=0.001$ and $P=0.002$, respectively). The mean and

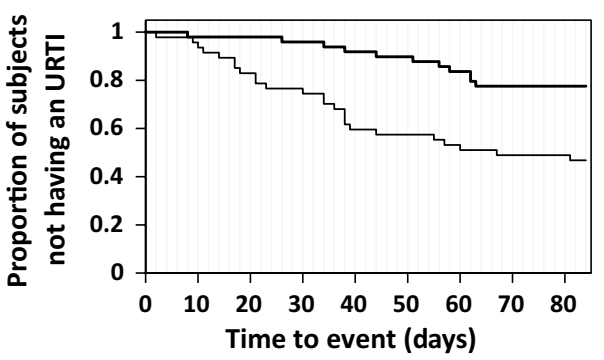

Fig. 2 Kaplan-Meier time-to-event curves for the first URTI. The URTI-free rates were 0.78 (95\% CI 0.66-0.89) and 0.47 (95\% CI $0.33-0.61$ ) in the LcS-FM (thick line) and the CM (thin line) groups, respectively

Table 3 Secondary outcomes: episode number per person, total days with symptoms per person, duration and severity of URTIs

\begin{tabular}{lcll}
\hline & $\begin{array}{l}\text { CM }(n=47) \\
\text { Mean (SD) }\end{array}$ & $\begin{array}{l}\text { LcS-FM }(n=49) \\
\text { Mean (SD) }\end{array}$ & $P$ value \\
\hline $\begin{array}{l}\text { Cumulative number of } \\
\text { URTI episodes }\end{array}$ & $0.7(0.7)$ & $0.3(0.8)$ & $0.004^{\mathrm{a}}$ \\
$\begin{array}{c}\text { Cumulative days with } \\
\text { symptoms (days) }\end{array}$ & $3.4(4.3)$ & $1.0(2.1)$ & $0.001^{\mathrm{b}}$ \\
$\begin{array}{c}\text { Duration per episode } \\
\text { (days) }\end{array}$ & $5.0(2.5)$ & $2.8(1.6)$ & $0.002^{\mathrm{b}}$ \\
$\begin{array}{l}\text { Mean severity score } \\
\text { Peak severity score }\end{array}$ & $15.8(2.8)$ & $15.9(2.6)$ & $0.966^{\mathrm{a}}$ \\
\hline
\end{tabular}

URTI upper respiratory tract infection, $C M$ control milk, $L c S-F M L$. casei strain Shirota-fermented milk

a $P$ values analyzed by the Wilcoxon two-sample test; $P<0.05$ is considered statistically significant

b $P$ values analyzed by the unpaired Student's $t$ test; $P<0.05$ is considered statistically significant

${ }^{c}$ Mean values of daily severity score during the period of each URTI episode

${ }^{\mathrm{d}}$ Peak values of daily severity score of URTIs

peak daily severity scores of URTIs did not differ between the two groups.

\section{Blood and saliva parameters}

Since immune defense mechanisms are important for host resistance to viral infections, we measured NK cell activity in peripheral blood mononuclear cells and $\operatorname{IgA}$ secretion rates in saliva at weeks 0,6 , and 12 of the intervention period. NK cell activity decreased at week 6 compared with week 0 in the CM group, but not in the LcS-FM group (Fig. 3). Thus, NK cell activity was significantly higher at week 6 in the LcS-FM group than in the CM group $(P=0.013)$. Salivary $\operatorname{IgA}$ secretion increased in weeks 6 and 12 in both groups, and the secretion did not differ between the groups. 

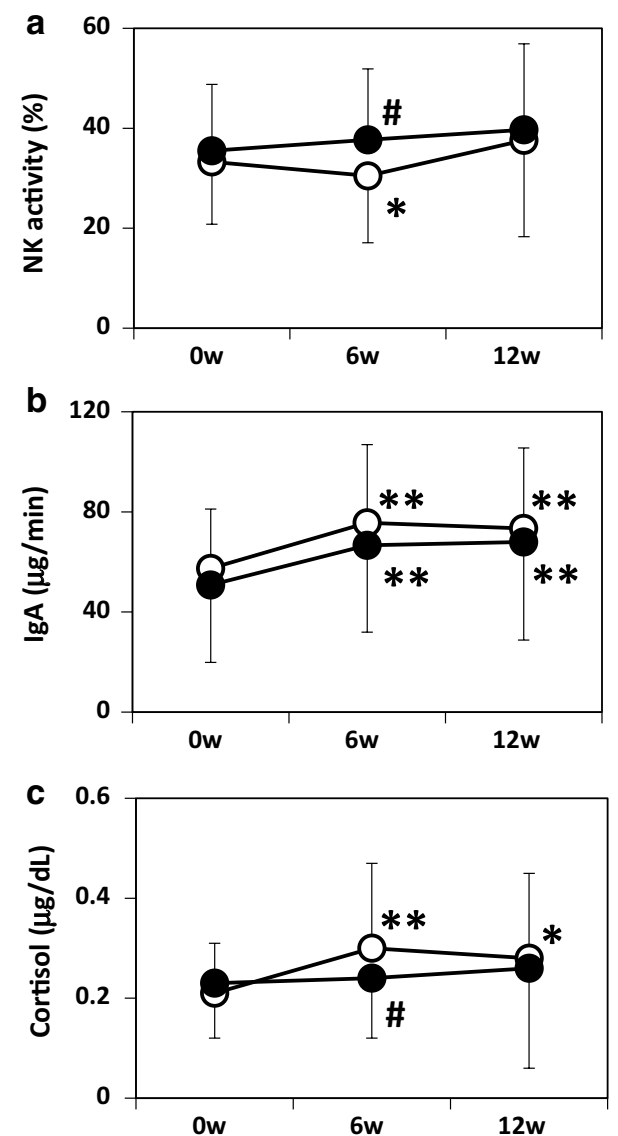

Fig. 3 Changes in NK cell activity in peripheral blood mononuclear cells, IgA secretion rate, and cortisol levels in saliva throughout the intervention period of 12 weeks. Data are expressed as means \pm SD. Open circles, CM group $(n=47)$; closed circles, LcS-FM group $(n=49)$. ${ }^{*} P<0.05 ; * * P<0.01$ versus week 0 in the same group; ${ }^{\#} P<0.05$ versus $\mathrm{CM}$ group

Salivary levels of the stress marker cortisol were analyzed. In contrast to the change in NK cell activity, cortisol levels were increased at week 6 in the CM group but not in the LcS-FM group, and the difference was significant between the groups $(P=0.045)$.

\section{Safety}

There were no adverse effects associated with consumption of the test drinks during the test period, as confirmed by the physician following examinations of blood and urine, physiological tests, analysis of health diaries, and interviews (data not shown).

\section{Discussion}

This randomized controlled trial conducted in healthy middle-aged male office workers clearly demonstrated that the daily intake of probiotic fermented milk, LcS-FM, reduced the risk of URTIs. The time to first URTI episode analysis showed that the risk reduction efficacy for LcS-FM was $68 \%$ (95\% CI 35-84\%) compared with CM. The analysis of URTI incidence for divided three periods showed that the positive health effects of LcS-FM were more evident in earlier test periods. The incidence of the common cold in the LcS-FM group (18.4\%) during the intervention period of 12 weeks decreased by more than half compared with the control group (44.7\%). The reduction in the incidence of influenza did not reach statistical significance, which might be due to the low incidence rates of both groups (10.6\% for the control; $4.1 \%$ for the LcS-FM group). In addition to the reduced risk for URTIs, LcS-FM reduced the cumulative number of days with URTI symptoms and shortened the duration of each episode.

Intervention studies using similar LcS-FM drinks against URTIs have been conducted previously. The results obtained from two previous trials in elderly participants aged over 80 years did not show a significant reduction in URTI incidence rates [24, 25]. In contrast, the present study clearly demonstrated that LcS-FM effectively reduced the risk of URTIs. These results are consistent with the results of a trial conducted in athletes aged $18-55$ years [20]. The ages of the participants in the latter study were similar to those in the present study, and thus, the different age groups among these studies might explain the conflicting results.

Another difference between the present and the previous studies should be noted. The viable number of LcS cells ingested daily was a minimum of $1.0 \times 10^{11}$ cells in this study, while in the aforementioned studies with elderly participants, the numbers of viable cells were $1.3 \times 10^{10}$ or $4.0 \times 10^{10}$. In general, a higher number of ingested viable probiotic cells will tend to lead to a more beneficial outcome. The dose-response associated with improved efficacy against URTIs has been reported. De Vrese et al. [28] showed that while the intake of a multivitamin and mineral tablet containing $5 \times 10^{7}$ viable lactobacillus and bifidobacterium cells shortened the duration of common cold infections, it had no effect on the incidence rate; however, another study showed that the similar tablet containing $5 \times 10^{8}$ viable probiotic cells reduced the incidence rate [29]. The relationship between viable LcS cell numbers and efficacy should be examined and discussed in future studies.

Many probiotic intervention studies that have demonstrated efficacy against URTIs have been conducted in infants, children, students, and the elderly [9-12]. Physically active individuals, including rugby players and athletes of endurance-based sports, might also be targets for probiotics [20, 27, 30]. Since immune defense activities are relatively weak in such populations, they generally experience more infections. The present study demonstrated that 
healthy middle-aged office workers are also a promising target population for probiotics. A clinical trial in employees at a manufacturing company in Sweden showed that daily consumption of $L$. reuteri ATCC 55730 reduced the incidence of sick leave due to respiratory or gastrointestinal illnesses during the 80 days of intervention [13]. The efficacy of probiotics was more evident in a subgroup of shift workers $[13,14]$. The present study demonstrated the efficacy of probiotics in typical desk workers. In general, such employees work under pressure and stress every day, which might be a risk factor for lowered immune defenses. Daily consumption of certain probiotics at higher doses may prevent disturbances in immune function resulting from stressful events in daily life. Thus, these results suggested the potential use of probiotics to improve health in the workplace.

In the present study, LcS-FM consumption led to improvements in immunological parameters and a stress marker. At week 6 of the intervention period, NK cell activity decreased and the salivary levels of cortisol increased in the control group, but these parameters kept within baseline levels in the LcS-FM group. NK cells play a very important role in the prevention of viral infections, including URTIs [15]. Inhibition of reduced NK cell activity might increase resistance to URTIs. The reason for the changes in immunological parameters and stress markers in the control group is not clear. However, the participants were assigned unusual daily tasks, including the recording of health information of many parameters during the intervention period, which might have affected their immune and endocrine systems, especially in the first half of the intervention period. At a later period, the participants might no longer feel such stress because getting used to the daily tasks and the parameters might return to the baseline levels. If the case is true, intake of LcS-FM may prevent decrease in immune defense activities caused by certain events, rather than augment their baseline levels. This may be the reason why improvements in the parameters by LcS-FM intake were detected only at week 6 . In fact, a previous study in healthy adults with the normal levels of NK cell activity showed that LcS intake had no effect on their NK cell activity [31].

Previous studies have revealed that the daily consumption of LcS-FM helps to recover low NK cell activity [18]. In vitro studies with human peripheral blood mononuclear cells suggest that LcS stimulates monocytes/macrophages to produce IL-12 and augments NK cell activity $[32,33]$. The direct regulation of immune function by LcS might be realized in the present clinical trial, which in turn might reduce the incidence of URTIs.

LcS-FM may mediate another protective mechanism whereby the ingestion of the probiotic drink reduces stress-related host responses. In the present study, LcSFM inhibited increases in the levels of the stress hormone cortisol, which has the ability to decrease NK cell activity [34]. Some clinical trials with probiotics or prebiotics have already focused on psychological stress-related decreases of host defense mechanisms. The prevention of URTIs by probiotic or prebiotic supplementation among university students under stress due to final exams was reported [35, 36]. However, in contrast to the present study, the levels of cortisol were not examined in these trials.

Increasing evidence suggests that probiotics and the intestinal microbiota affect the nervous system and brain function [37]. Recently, Tanida et al. [38, 39] showed that some probiotic strains including $\mathrm{LcS}$ suppress the neural activity of sympathetic nerves in rats. The nervous system is closely related to the immune system [40]. Future studies should focus on nervous system-related actions of probiotics as well as their direct immune modulatory activities to obtain a better understanding of the precise mechanisms of preventing viral infections via probiotics.

In conclusion, the findings obtained in this study suggest that the daily intake of fermented milk with LcS may reduce the risk of URTIs in healthy middle-aged office workers, probably through modulation of the immune system.

Acknowledgments We thank the staff of the Creative Pioneer for Clinical Challenge Co Ltd (Tokyo, Japan) for their help with participant management, sample analysis, and data analysis. We acknowledge Dr. Mitsuhisa Kawai, the Yakult Central Institute, for the calculation of sample size and the statistical analyses. We also thank the participants in this study. This study received no specific grant from any funding agency.

\section{Compliance with ethical standards}

Conflict of interest All of the authors are employed by the Yakult Honsha, which produces fermented dairy products using the probiotic strain LcS.

Open Access This article is distributed under the terms of the Creative Commons Attribution 4.0 International License (http://creativecommons.org/licenses/by/4.0/), which permits unrestricted use, distribution, and reproduction in any medium, provided you give appropriate credit to the original author(s) and the source, provide a link to the Creative Commons license, and indicate if changes were made.

\section{References}

1. Neish AS (2009) Microbes in gastrointestinal health and disease. Gastroenterology 136:65-80

2. Fuller R (1989) Probiotics in man and animals. J Appl Bacteriol 66:365-378

3. Shida K, Nanno M (2008) Probiotics and immunology: separating the wheat from the chaff. Trends Immunol 29:565-573

4. Mitsuoka T (2014) Development of functional food. Biosci Microbiota Food Health 33:117-128

5. Nomoto K (2005) Prevention of infections by probiotics. J Biosci Bioeng 100:583-592 
6. Alvarez-Olmos MI, Oberhelman RA (2001) Probiotic agents and infectious diseases: a modern perspective on a traditional therapy. Clin Infect Dis 32:1567-1576

7. King S, Glanville J, Sanders ME, Fitzgerald A, Varley D (2014) Effectiveness of probiotics on the duration of illness in healthy children and adults who develop common acute respiratory infectious conditions: a systematic review and meta-analysis. $\mathrm{Br}$ J Nutr 112:41-54

8. Hao Q, Lu Z, Dong BR, Huang CQ, Wu T (2015) Probiotics for preventing acute upper respiratory tract infections. Cochrane Database Syst Rev 2:CD006895. doi:10.1002/14651858. CD006895.pub3

9. Taipale T, Pienihakkinen K, Isolauri E, Larsen C, Brockmann E, Alanen P, Jokela J, Soderling E (2011) Bifidobacterium animalis subsp. lactis BB-12 in reducing the risk of infections in infancy. Br J Nutr 105:409-416

10. Merenstein D, Murphy M, Fokar A, Hernandez RK, Park H, Nsouli H, Sanders ME, Davis BA, Niborski V, Tondu F, Shara NM (2010) Use of a fermented dairy probiotic drink containing Lactobacillus casei (DN-114 001) to decrease the rate of illness in kids: the DRINK study. A patient-oriented, double-blind, cluster-randomized, placebo-controlled, clinical trial. Eur J Clin Nutr 64:669-677

11. Waki N, Matsumoto M, Fukui Y, Suganuma H (2014) Effects of probiotic Lactobacillus brevis KB290 on incidence of influenza infection among schoolchildren: an open-label pilot study. Lett Appl Microbiol 59:565-571

12. Makino S, Ikegami S, Kume A, Horiuchi H, Sasaki H, Orii N (2010) Reducing the risk of infection in the elderly by dietary intake of yoghurt fermented with Lactobacillus delbrueckii ssp. bulgaricus OLL1073R-1. Br J Nutr 104:998-1006

13. Tubelius P, Stan V, Zachrisson A (2005) Increasing work-place healthiness with the probiotic Lactobacillus reuteri: a randomised, double-blind placebo-controlled study. Environ Health $4: 25$

14. Guillemard E, Tanguy J, Flavigny A, de la Motte S, Schrezenmeir J (2010) Effects of consumption of a fermented dairy product containing the probiotic Lactobacillus casei DN-114 001 on common respiratory and gastrointestinal infections in shift workers in a randomized controlled trial. J Am Coll Nutr 29:455-468

15. Biron CA, Nguyen KB, Pien GC, Cousens LP, Salazar-Mather TP (1999) Natural killer cells in antiviral defense: function and regulation by innate cytokines. Annu Rev Immunol 17:189-220

16. Neville V, Gleeson M, Folland JP (2008) Salivary IgA as a risk factor for upper respiratory infections in elite professional athletes. Med Sci Sports Exerc 40:1228-1236

17. Morimoto K, Takeshita T, Inoue-Sakurai C, Maruyama S (2001) Lifestyles and mental health status are associated with natural killer cell and lymphokine-activated killer cell activities. Sci Total Environ 270:3-11

18. Nanno M, Kato I, Kobayashi T, Shida K (2011) Biological effects of probiotics: what impact does Lactobacillus casei Shirota have on us? Int J Immunopathol Pharmacol 24:45S-50S

19. Kotani Y, Shinkai S, Okamatsu H, Toba M, Ogawa K, Yoshida H, Fukaya T, Fujiwara Y, Chaves PH, Kakumoto K, Kohda N (2010) Oral intake of Lactobacillus pentosus strain b240 accelerates salivary immunoglobulin A secretion in the elderly: a randomized, placebo-controlled, double-blind trial. Immun Ageing 7:11

20. Gleeson M, Bishop NC, Oliveira M, Tauler P (2011) Daily probiotic's (Lactobacillus casei Shirota) reduction of infection incidence in athletes. Int J Sport Nutr Exerc Metab 21:55-64

21. Matsumoto K, Takada T, Shimizu K, Moriyama K, Kawakami K, Hirano K, Kajimoto O, Nomoto K (2010) Effects of a probiotic fermented milk beverage containing Lactobacillus casei strain Shirota on defecation frequency, intestinal microbiota, and the intestinal environment of healthy individuals with soft stools. J Biosci Bioeng 110:547-552

22. Nanno M, Matsumoto S, Shida S (2012) Lactobacillus casei strain Shirota: benefits based on a long history of usage. In: Nair GB, Takeda Y (eds) Health impact of probiotics. Elsevier, New Delhi, pp 85-98

23. Shida K, Nomoto K (2013) Probiotics as efficient immunopotentiators: translational role in cancer prevention. Indian J Med Res 138:808-814

24. Fujita R, Iimuro S, Shinozaki T, Sakamaki K, Uemura Y, Takeuchi A, Matsuyama Y, Ohashi Y (2013) Decreased duration of acute upper respiratory tract infections with daily intake of fermented milk: a multicenter, double-blinded, randomized comparative study in users of day care facilities for the elderly population. Am J Infect Control 41:1231-1235

25. Van Puyenbroeck K, Hens N, Coenen S, Michiels B, Beunckens C, Molenberghs G, Van Royen P, Verhoeven V (2012) Efficacy of daily intake of Lactobacillus casei Shirota on respiratory symptoms and influenza vaccination immune response: a randomized, double-blind, placebo-controlled trial in healthy elderly nursing home residents. Am J Clin Nutr 95:1165-1171

26. Berggren A, Lazou Ahren I, Larsson N, Onning G (2011) Randomised, double-blind and placebo-controlled study using new probiotic lactobacilli for strengthening the body immune defence against viral infections. Eur J Nutr 50:203-210

27. West NP, Horn PL, Pyne DB, Gebski VJ, Lahtinen SJ, Fricker PA, Cripps AW (2013) Probiotic supplementation for respiratory and gastrointestinal illness symptoms in healthy physically active individuals. Clin Nutr 33:581-587

28. de Vrese M, Winkler P, Rautenberg P, Harder T, Noah C, Laue C, Ott S, Hampe J, Schreiber S, Heller K, Schrezenmeir J (2006) Probiotic bacteria reduced duration and severity but not the incidence of common cold episodes in a double blind, randomized, controlled trial. Vaccine 24:6670-6674

29. Winkler P, de Vrese M, Laue C, Schrezenmeir J (2005) Effect of a dietary supplement containing probiotic bacteria plus vitamins and minerals on common cold infections and cellular immune parameters. Int J Clin Pharmacol Ther 43:318-326

30. Haywood BA, Black KE, Baker D, McGarvey J, Healey P, Brown RC (2014) Probiotic supplementation reduces the duration and incidence of infections but not severity in elite rugby union players. J Sci Med Sport 17:356-360

31. Spanhaak S, Havenaar R, Schaafsma G (1998) The effect of consumption of milk fermented by Lactobacillus casei strain Shirota on the intestinal microflora and immune parameters in humans. Eur J Clin Nutr 52:899-907

32. Shida K, Suzuki T, Kiyoshima-Shibata J, Shimada S, Nanno M (2006) Essential roles of monocytes in stimulating human peripheral blood mononuclear cells with Lactobacillus casei to produce cytokines and augment natural killer cell activity. Clin Vaccine Immunol 13:997-1003

33. Takeda K, Suzuki T, Shimada SI, Shida K, Nanno M, Okumura K (2006) Interleukin-12 is involved in the enhancement of human natural killer cell activity by Lactobacillus casei Shirota. Clin Exp Immunol 146:109-115

34. Mavoungou E, Bouyou-Akotet MK, Kremsner PG (2005) Effects of prolactin and cortisol on natural killer (NK) cell surface expression and function of human natural cytotoxicity receptors (NKp46, NKp44 and NKp30). Clin Exp Immunol 139:287-296

35. Langkamp-Henken B, Rowe CC, Ford AL, Christman MC, Nieves C Jr, Khouri L, Specht GJ, Girard SA, Spaiser SJ, Dahl WJ (2015) Bifidobacterium bifidum R0071 results in a greater proportion of healthy days and a lower percentage of academically stressed students reporting a day of cold/flu: a 
randomised, double-blind, placebo-controlled study. Br J Nutr 113:426-434

36. Hughes C, Davoodi-Semiromi Y, Colee JC, Culpepper T, Dahl WJ, Mai V, Christman MC, Langkamp-Henken B (2011) Galactooligosaccharide supplementation reduces stress-induced gastrointestinal dysfunction and days of cold or flu: a randomized, double-blind, controlled trial in healthy university students. Am J Clin Nutr 93:1305-1311

37. Wang Y, Kasper LH (2014) The role of microbiome in central nervous system disorders. Brain Behav Immun 38:1-12

38. Tanida M, Nagai K (2011) Electrophysiological analysis of the mechanism of autonomic action by lactobacilli. Biosci Microflora 30:99-109
39. Tanida M, Imanishi K, Akashi H, Kurata Y, Chonan O, Naito E, Kunihiro S, Kawai M, Kato-Kataoka A, Shibamoto T (2014) Injection of Lactobacillus casei strain Shirota affects autonomic nerve activities in a tissue-specific manner, and regulates glucose and lipid metabolism in rats. J Diabetes Investig 5:153-161

40. Segerstrom SC, Miller GE (2004) Psychological stress and the human immune system: a meta-analytic study of 30 years of inquiry. Psychol Bull 130:601-630 